\title{
Development of a Flexible Pressure Sensor for Measurement of Endotension
}

\author{
Isa C. T. Santos \& João Manuel R. S. Tavares \\ Instituto de Engenharia Mecânica e Gestão Industrial / Faculdade de Engenharia, Universidade do Porto
}

PORTUGAL

\author{
A.T. Sepúlveda, A.J. Pontes, J.C. Viana \& L.A. Rocha \\ Institute for Polymers and Composites/I3N, University of Minho
}

PORTUGAL

ABSTRACT: An aneurysm is a bulge in a weakened portion of a blood vessel wall much like the bulge that results from over-inflating an inner tube. If left untreated, it may burst or rupture causing shock and/or death due to massive blood loss. Endovascular aneurysm repair (EVAR) is one of the treatments available for aortic aneurysms but, in spite of major advances in the operating techniques, complications still occur and lifelong surveillance is recommended. Current surveillance protocols are based on medical imaging exams that besides being expensive are time consuming. After a brief introduction to EVAR and its complications, this paper reviews post-EVAR surveillance protocols and the current devices to measure endotension. Finally, are introduced two new concepts for a flexible pressure sensor with passive telemetry.

\section{INTRODUCTION}

An aneurysm is a bulge in a weakened portion of a blood vessel wall, Figure 1. It can be defined as a permanent and irreversible localized dilatation of an artery, having at least a $50 \%$ increase in diameter compared with the common one (Johnston et al. 1991).
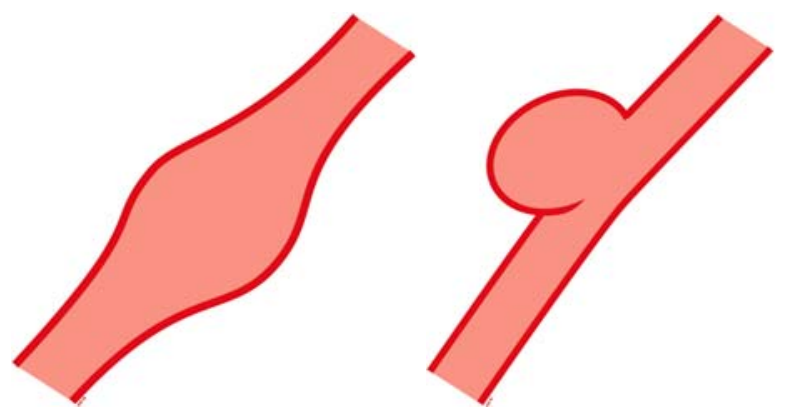

Figure 1: Representation of a fusiform aneurysm (on the left) and a saccular aneurysm (on the right).

An aortic aneurysm, Figure 2, if left untreated may burst or rupture causing shock, and even death, due to massive blood loss. Endovascular aneurysm repair (EVAR) is one of the treatments available for this serious disease. It is a minimally invasive procedure in which a stent-graft, Figure 3, is guided from the femoral artery to the affected artery segment to prevent aneurysm rupture by exclusion of the aneurysm sac from systemic pressure. Since it was proposed by Parodi (Parodi et al. 1991), in the early 1990's, has become widely accepted due to advantages such as decreased blood loss, early morbidity and mortality, shorter hospitalization
(Ricotta II et al. 2009), reduced patient discomforts and potentially lower costs.

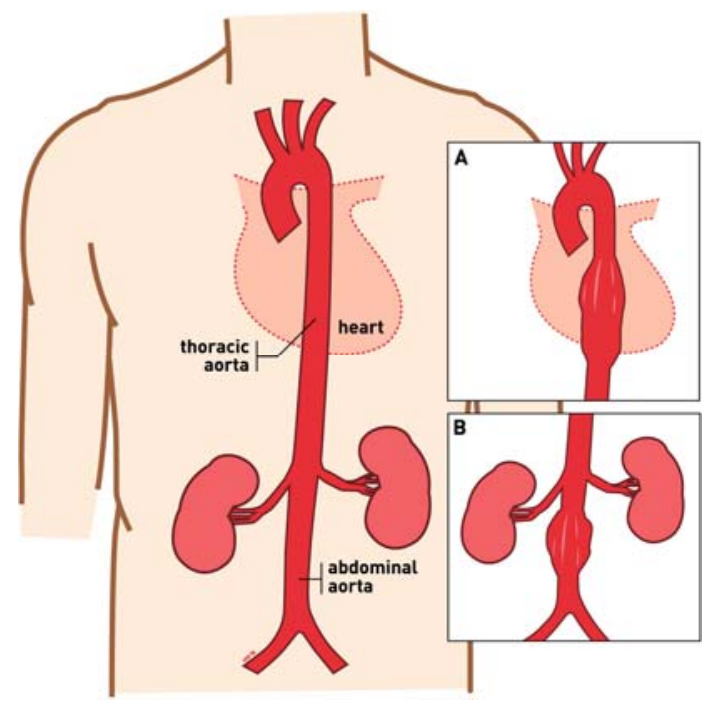

Figure 2: Representation of a normal aorta, (A) a thoracic aortic aneurysm (TAA), and (B) an abdominal aortic aneurysm (AAA).

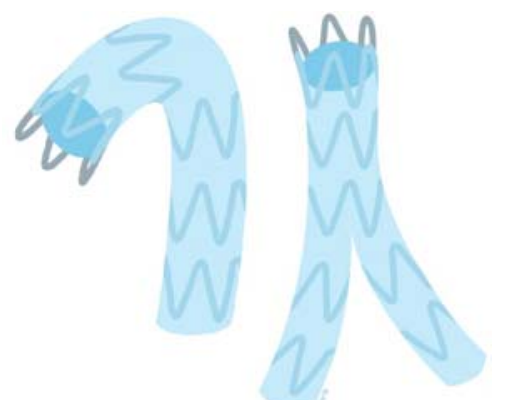

Figure 3: Representation of a thoracic stent-graft (on the left) and an abdominal stent-graft (on the right). 
Albeit major advances in EVAR techniques, complications still occur and lifelong surveillance is recommended (Baril et al. 2007).

Presently, follow-up protocols are based in imaging exams that aim to evaluate the size of the aneurysm sac, detect endoleaks, endograft migration, module disconnection, or component fatigue and failure (stent fracture, graft tears). The medical imaging exams provide great information about aneurysm exclusion and sac morphology but do not provide any direct measurement of pressure within the aneurysm sac (Baril et al. 2007).

This paper presents the development of a novel pressure sensor for the measurement of endotension. Its distinctive feature is flexibility, which will allow the conformability of the sensor to the stent-graft and thus the aorta. Such device, in comparison with others, can be attached to the stent-graft and delivered in a single procedure. Furthermore, it enables the placement of more than one sensor, a sensor cluster, contributing to a better posendovascular aneurysm study that so far has not been possible.

After an introduction to the topic, EVAR complications will be identified as well as current surveillance protocols. Next, a review on the existing devices and methods for the measurement of the aneurysm sac pressure will be presented. Finally, the development of the new sensor will be considered.

\section{EVAR COMPLICATIONS}

EVAR has a unique set of possible complications, which occur at a pertinent rate (Ricotta II et al. 2009). However, as surgeons have gained experience with the procedure, the frequency of complications has decreased. Furthermore, it must be taken into account that all devices are subjected to these possible complications, i.e., they are not exclusive of a specific device.

EVAR complications can be described as early, if they occur less than 30 days after the procedure, or as late, otherwise. They can be further categorized as being related to the delivery, the deployment, the devices, systemic problems, and operator errors (Katzen et al. 2006). Table 1 summarizes the complications concerning the deployment and the device.

Stent-graft migration refers to an inappropriate movement of the device. It can be explained by the failure of the attachment with an unchanged aorta or as a failure related to changes in aneurysm morphology. This complication can be prevented over sizing the endoprosthesis to promote friction and/or barb or hook penetration. Other factors that may contribute to fixation of the device include suprarenal attachment, column strength, and arterial ingrowth (Li et al. 2006).
Table 1. Complications specific to endovascular grafts (adapted from (Katzen et al. 2006)).

\begin{tabular}{ll}
\hline \multicolumn{1}{c}{ Early } & \multicolumn{1}{c}{ Late } \\
\hline Graft kink & Graft migration \\
Endoleaks & Neck dilatation \\
Stent-graft structural failure & Sac enlargement \\
Graft infection & Endoleaks \\
& Endotension \\
& Graft tear or failure \\
& Material fatigue \\
& Stent breakage \\
& Component separation \\
\hline
\end{tabular}

Endoleak is a term that describes persistent aneurysm sac perfusion and pressurization (Katzen et al. 2006). It is the most common complication after stent-graft implantation (Mita et al. 2000) and occur mainly due to incomplete seal of the endovascular graft. Endoleaks have been categorized as follows in Table 2 and are treated by a variety of means, including conversion to surgical repair, or insertion of a new stent or graft.

Table 2. Classification of endoleaks (adapted from (Katzen et al. 2006)).

\begin{tabular}{ll}
\multicolumn{1}{c}{ Type } & \multicolumn{1}{c}{ Description } \\
\hline I & Attachment site leaks \\
II & Branch leaks (without attachment site connection) \\
III & Graft defect \\
IV & Graft wall (fabric) porosity \\
\hline
\end{tabular}

Endotension is defined as increased pressure within the sac without the presence of an endoleak. This condition may result from the accumulation of fluid within the aneurysm sac or due to the transmission through the wall of the endograft, around its ends at the attachment zones (White 2001). Its consequences are unknown so far.

\section{SURVEILLANCE PROTOCOLS AFTER EVAR} Nowadays, imaging exams, such as computed tomography (CT) scan, and magnetic resonance angiography (MRA), are the first choice examination to identify inadequate aneurysm exclusion, device migration, and secondary markers of sac pressurization, namely aneurysm expansion and endoleaks.

The standard surveillance protocol involves exams at 1, 6, and 12 months after the EVAR procedure, and thereafter, on an annual basis (Milner et al. 2006).

Device migration and stent fractures or other indication of device fatigue are clear in plain abdominal radiography. Ultrasonography allows the measurement of the aneurysm sac and is effective in the detection of endoleaks but, even with enhanced sensitivity obtained with the use of contrast agents, requires a skilled technician to interpret the exams. CTA (computed tomography angiography), MRI (magnetic resonance imaging) and MRA are sensitive tools to detect endoleaks but cannot be 
repeated often due to radiation and/or the use of nephrotoxic contrast agents. Furthermore, these exams can be considered time consuming and expensive.

\section{MEASUREMENT OF ANEURYSM SAC PRESSURE}

Published data describe the use of catheters to measure pressure in the residual aneurysm sac (Carnero et al. 2007). However, although these methods provide precise measurements (Baril et al. 2007), they are invasive and bear multiple risks.

An alternative method for the measurement of the aneurysm sac's pressure is the implant of remote pressure transducers during EVAR. This solution is advantageous since measurements can be done as needed (hourly, weekly, etc.) in the patient's home or office instead of a hospital once or twice a year. Another important feature is the fact of, without risks for the patients, being possible to measure both the mean pressure and the pulsatile pressure. Currently, three telemetric pressure sensors are available: the Impressure AAA Sac Pressure Transducer or RemonAAA (Remon Medical, Tel Aviv, Israel), the EndoSure Wireless Pressure Sensor (CardioMEMS, Inc, Atlanta, USA) and, the TPS Telemetric Pressure Sensor developed by the Helmholtz-Institute for Biomedical Engineering, RWTH Aachen in cooperation with the Institute of Materials in Electrical Engineering, RWTH Aachen (Springer et al. 2007a).

The Remon Impressure AAA Sac Pressure Transducer measures $3 \mathrm{~mm} \times 9 \mathrm{~mm} \times 1.5 \mathrm{~mm}$ and is sewn to the outside of the stent-graft, which is then repackaged in the delivery sheath. It consists of a piezoelectric membrane that when actuated by ultrasound waves from a hand-held probe charges a capacitor. Once charged, the transducer measures ambient pressure, then generates an ultrasound signal, which is relayed to the probe. The data can be downloaded and exported as an Excel data file consisting of pressure measurements and the corresponding times at which the measurements were taken (Ellozy et al. 2004).

The EndoSure Wireless Pressure Sensor measures approximately $30 \mathrm{~mm} \times 5 \mathrm{~mm} \times 1.5 \mathrm{~mm}$ and is delivered into the aneurysm sac through its own sheath (completely separate from the aortic endograft). It is made by laminating together several layers to form a capacitor. Metal spirals in the first and last layer form the inductor components of an electrical circuit. Current induction in the sensor results in energy oscillation that varies with frequency. Changes in the circuit's resonant frequency are directly proportional to the force applied to the sensor's surface (in this case, the pressure within the aneurysm sac). The inductor allows electromagnetic coupling between the sensor and the electronic system. The latter consists of an antenna held against the patient's side or back in the area where the sensor is located; it measures the resonant frequency, which is then displayed on a computer screen (Silveira et al. 2008).

The TPS Telemetric Pressure Sensor consists of an implantable sensor capsule and an external readout station. The capsule comprises a capacitive absolute pressure sensor and an in-capsule signalprocessing microchip including an inductive telemetry unit. Like the other devices previously described, does not require an internal power source.

The TPS measures $26 \mathrm{~mm}$ in length and $3.3 \mathrm{~mm}$ in diameter. It has fixation holes at both ends allowing to either suture to the outer wall of the endovascular prosthesis before the EVAR procedure and deployed as a complete system or introduced separately through a regular 11 French catheter system and stabilized within the aneurysm sac, using e.g. self-inflating wires (Springer et al. 2007b).

\section{DEVELOPMENT OF A NOVEL SENSOR}

A new flexible pressure sensor with passive telemetry to be integrated in a stent graft is currently under development by the authors. The focus is on the use of a flexible substrate enabling the conformability of the sensor to the stent-graft and thus the aorta.

Given the characteristics of the application (the sensor will be attached to the stent-graft) the capacitive sensor must be foldable, extremely flexible and characterized by a very small profile. In addition, the technology should be simple and biocompatible. Silicon based microtechnologies are widely used in implantable medical devices (Receveur et al. 2007), but due to the application specifications, a new fabrication process is being developed.

The pressure monitoring system uses a passive telemetry system, based on an implantable LC resonant network, for the external readout of the pressure sensor signal. The use of passive telemetry in implantable medical devices is common (Mokwa 2007) and enables the realization of active implants with no power constraints.

The fabrication of the implantable pressure monitoring system (capacitive sensor and inductor) is being pursued using two different approaches, both based on a thin flexible substrate made of polydimethylsiloxane (PDMS) and constructed through the use of acrylic molds. While the first approach uses nano-engineered Aligned Carbon Nano-Tubes (ACNT) for the implementation of the passive electronic components, the second uses inkjet printed conductive inks. Figures 4 and 5 show the process flow for each of these approaches.

Both processes start with the production of acrylic molds, using CNC milling, for posterior 
fabrication of the PDMS membranes. This technique has low costs and fast production times, but it is associated with poor dimensional control.

In the case of the CNT based approach, Chemical Vapor Deposition (CVD) is used to growth forests or "carpets" of vertically-aligned CNTs (Bello et al. 2008). A silicon substrate with patterned $\mathrm{Fe} / \mathrm{Al}_{2} \mathrm{O}_{3}$ catalyst is placed on a horizontal quartz tube furnace at atmospheric pressure at $750{ }^{\circ} \mathrm{C}$ for the CNTs growth. This method has the advantage of allowing the growth of high purity, high yield and vertically aligned CNTs.

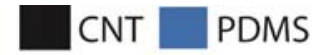

a)

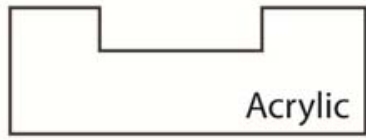

Mold Fabrication

b)

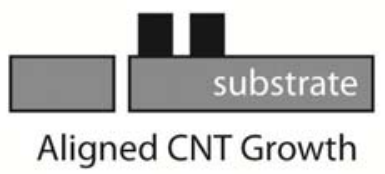

c)

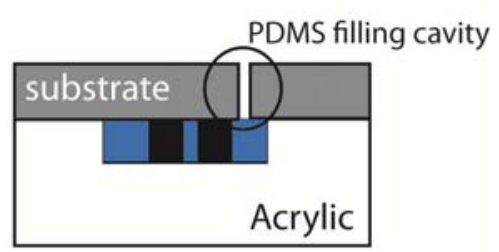

PDMS Filling

d)

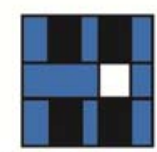

PDMS Bonding

Figure 4: Fabrication process flow for the development of a flexible pressure sensor using CNTs.

Afterward, the CNTs are embedded into the polymer matrix (PDMS). This step is schematically represented in Figure 4c. The substrate with the CNTs is placed against the moulds, and the PDMS is introduced in the cavities through a hole, followed by the curing of the elastomer. A similar process is used in the case of the second approach, to produce the PDMS membranes for posterior inkjet printing of the conductive inks. The main concern here is the adhesion between the inks and the PDMS, which may require surface treatment of the PDMS membranes.

Finally, and since the flexible pressure sensor is composed of three thin layers, with the top and bottom layers defining the inductor and the electrodes, and the middle one defining the dielectric (air), a bonding step is performed. The PDMS membranes are bonded using uncured PDMS adhesive techniques.

Both methodologies present several challenges. The use of ACNT technology requires improvements in the growth control of the carbon nano-tubes and enhancement of the electric conductivity of ACNT embedded polymeric matrixes. Printing the components requires developments on conductive inks, in order to achieve enough conductivity and adherence to PDMS.

a)

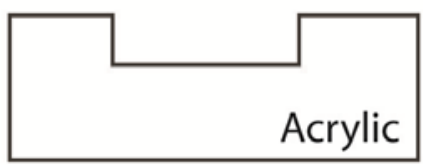

Mold Fabrication

b)

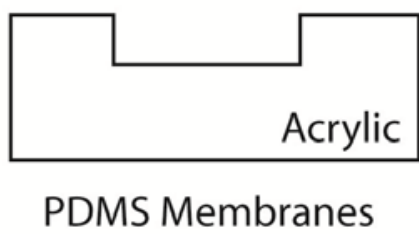

c)

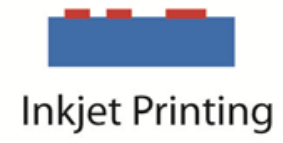

d)

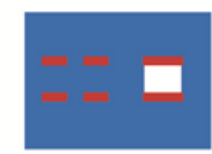

PDMS Bonding

\section{Conductive Ink PDMS}

Figure 5: Fabrication process flow for the development of a flexible pressure sensor using conductive inks.

In both cases, the mechanical and electric behaviors of the flexible film need further studies.

\section{CONCLUSION}

Although medical imaging exams provide ample information regarding the aneurysm exclusion and the sac morphology, they are time consuming and expensive. As an alternative method, implantable remote pressure transducers have been developed. We described the ImPressure AAA Sac Pressure Sensor (the first implanted pressure sensor used in animal models and a small clinical trial), the EndoSure Wireless AAA Pressure Sensor (the only pressure sensor with FDA approval) and, the TPS Telemetric Pressure Sensor (based on a completely digital data-processing and transmitting unit). Finally, we introduced a novel flexible pressure 
sensor with passive telemetry that is underdevelopment.

\section{ACKNOWLEDGMENTS}

The first author wishes to thank FCT - Fundação para a Ciência e Tecnologia, in Portugal, for the financial support provided by the grant SFRH/BD/42967/2008.

This work is supported by FCT under the project MIT-Pt/EDAM-EMD/0007/2008.

\section{REFERENCES}

Baril, D.T., Kahn, R. A. \& et al. 2007. Endovascular abdominal aortic aneurysm repair: Emerging developments and anesthetic considerations. Journal of Cardiothoracic and Vascular Anesthesia 21(5): 730-742.

Bello, D., A. J. Hart \& et al. 2008. Particle exposure levels during CVD growth and subsequent handling of vertically-aligned carbon nanotube films. Carbon 46(6): 974-977.

Carnero, L. \& Milner R. 2007. Advanced endovascular therapy of aortic disease. Blackwell Publishing

Ellozy, S. H., A. Carroccio \& et al. 2004. First experience in human beings with a permanently implantable intrasac pressure transducer for monitoring endovascular repair of abdominal aortic aneurysms. Journal of Vascular Surgery 40(3): 405-412.

Johnston, K. W., B. R. Robert \& et al. 1991. Suggested standards for reporting on arterial aneurysms. Journal of Vascular Surgery 13(3): 452-458.

Katzen, B.T. \& MacLean A.A. 2006. Complications of endovascular repair of abdominal aortic aneurysms: A review. CardioVascular and Interventional Radiology 29(6): 935-946.

Li, Z. \& C. Kleinstreuer 2006. Analysis of biomechanical factors affecting stent-graft migration in an abdominal aortic aneurysm model. Journal of Biomechanics 39(12): 2264-2273.

Milner, R., Kasirajan, K. \& et al. 2006. Future of endograft surveillance. Seminars in Vascular Surgery 19(2): 7582.

Mita, T., T. Arita \& et al. 2000. Complications of endovascular repair for thoracic and abdominal aortic aneurysm: An imaging spectrum. Radiographics 20(5): 1263-1278.

Mokwa, W. 2007. Medical implants based on microsystems. Measurement Science and Technology 18(5): R47.

Parodi, J.C., Palmaz J.C., \& et al. 1991. Transfemoral intraluminal graft implantation for abdominal aortic aneurysms. Annals of Vascular Surgery 5(6): 491-499.

Receveur, R. A. M., F. W. Lindemans \& et al. 2007. Microsystem technologies for implantable applications. Journal of Micromechanics and Microengineering 17(5): R50.

Ricotta II, J.J., Malgor, R.D. \& et al. 2009. Endovascular abdominal aortic aneurysm repair: Part I. Annals of Vascular Surgery 23(6): 799-812.

Silveira, P. G., C. W. T. Miller \& et al. 2008. Correlation between intrasac pressure measurements of a pressure sensor and an angiographic catheter during endovascular repair of abdominal aortic aneurysm. Clinics 63: 59-66.

Springer, F., Günther, R.W. \& et al. 2007a. Aneurysm sac pressure measurement with minimally invasive implantable pressure sensors: An alternative to current surveillance regimes after EVAR?. CardioVascular and Interventional Radiology 31(3): 460-467.

Springer, F., R. Schlierf \& et al. 2007b. Detecting endoleaks after endovascular AAA repair with a minimally invasive, implantable, telemetric pressure sensor: an in vitro study. European Radiology 17(10): 25892597.

White, G. H. 2001. What Are the Causes of Endotension? Journal of Endovascular Therapy 8(5): 454-456. 